\title{
CHOREO-ATHETOID AND CHOREOPSYCHOTIC SYN- DROMES AS CLINICAL TYPES OR SEQUELAE OF EPIDEMIC ENCEPHALITIS *
}

\author{
LASALLE ARCHAMBAULT, M.D.
}

ALBANY, N. Y.

Epidemic encephalitis, also known as lethargic encephalitis, nona, infectious ophthalmoplegia and by various other designations, has only recently sprung into prominence but by reason of its rapid spread over the greater part of both hemispheres in less than two years, it has engrossed to an unparalleled degree the attention of scientists throughout the world. 'Although von Economo's first description of this disease only dates back to the spring of 1917 the amount of literature concerning it has already reached incredible proportions, a fact which has served promptly to vulgarize the symptomatology and diagnostic features of this newly isolated affection and also to provide us with a preliminary knowledge of the character and distribution of the underlying histologic changes.

It seems well established that the lesions involve with especial predilection the tegmentum of the pontopeduncular region and the basal ganglions, and give rise clinically, in the majority of cases, to a fairly distinctive symptomatic picture characterized essentially by fever, progressive lethargy and asthenia, ocular and facial palsies, more or less generalized rigidity or passivity. and occasional myoclonic twitchings. The frequent occurrence, however, of quite dissimilar though almost equally typical syndromes affords presumptive evidence that in this disease the histologic changes may be disseminated through the entire nervous system with focal predominance at any given level. It probably will be shown in time that, much in the same fashion as in poliomyelitis, the changes in epidemic encephalitis are generalized in the great majority of cases and that what is now regarded as the classic clinical physiognomy of the disease simply depends on the commonly overwhelming preponderance of the lesions in the periventricular gray matter compassed by the striatothalamotegmental region. This is indeed the favorite localization of the morbid process in epidemic encephalitis and its clinical expression has been so abundantly studied and widely depicted that it can hardly escape detection. It is just as important to recognize the more unusual or atypical forms of the disease, though, be it stated at once, they are atypical only

* Read at the Forty-Sixth Annual Meeting of the American Neurological Association, New York, June, 1920. 
by contrast with the more familiar clinical picture sketched in the foregoing. In reality they represent various aberrant or level types in which the salient clinical features are so strikingly foreign to the usual cardinal grouping that diagnosis is frequently jeopardized. Many clinical types of epidemic encephalitis have already been encountered and described and the constant report in the literature of additional syndromes bids fair to extend almost indefinitely the possibility of revised classification of the numerous forms or syndromes to which the remarkably protean symptomatology of the disease may give rise.

Several authors have already hazarded a practical tabulation of the various clinical types of epidemic encephalitis, and to some such a classification at this time may, perhaps, seem somewhat premature, but when one fully realizes the not infrequent difficulties in differential diagnosis which arise in connection with certain forms resembling alcoholic delirium or general paresis, cerebral tumor or cerebral abscess, acute meningitis or poliomyelitis, vascular lesions of the cerebellum, etc., any attempt at early classification will be conceded to be both a judicious and laudable effort. Furthermore, it is no idle task to utilize the valuable and suggestive data furnished by the variable clinical aspects of epidemic encephalitis in a legitimate endeavor to elucidate many obscure problems of cerebral physiology and localization. There can be no doubt that the intensive study, both anatomic and clinical, of this newly identified cerebrospinal affection promises to shed much light on the pathogenesis of several hitherto baffling neurologic disorders such as paralysis agitans, choreiform and athetoid states, endocrinal dysfunctions and even certain psychoses and psychoneuroses.

.The various clinical forms of epidemic encephalitis thus far signalized include the following types: lethargic, cataleptic, polioencephalitic, anterior and posterior poliomyelitis, Parkinsonian, acute psychotic, epileptomaniacal, meningitic, meningoradicular, polyneuritic, cerebellar, hemiplegic, diplegic, monoplegic, as well as a form occurring in infants and called for this reason encephalitis neonatorum. In reality there are still other types, and by the time the chapter devoted to this disease has been fully written we probably will find that it embraces clinical syndromes that reproduce with surprising precision the outstanding features of many of the known acute and subacute diseases of the nervous system.

A point which cannot be too strongly emphasized is that aside from a classification based on predominating clinical features and intended to establish the existence of level types, a classification based on the course or evolution of the disease must likewise receive due consideration. Some authors regard the disease as having essentially an acute 
onset, while others describe the onset as subacute or insidious. The fact seems to be that both modes of onset are about equally common. The duration of the disease is extremely variable, stated by most observers as being approximately eight weeks in the majority of cases. A hyperacute or fulminating course is extremely rare, yet I have seen two undoubted cases of epidemic encephalitis terminate fatally in less than a week with acute bulbar paralysis. It is becoming more and more evident likewise that a very prolonged or chronic course extending over several months is far from uncommon and this fact is of the greatest importance in explaining the frequent relapses or the recrudescence of certain symptoms observed in patients who, because of the relatively mild and purely evanescent character of their initial disturbances, have been prematurely discharged from hospital service or allowed to resume work. This same factor of chronicity affords an explanation of certain unusual syndromes which soon follow the complete disappearance of a few abortive manifestations of the usual type of the disease and even continue to develop in the pseudoconvalescent stage until a fairly well defined parkinsonian or choreoathetoid syndrome is materialized. Under these circumstances we are apt to interpret such developments as sequelae of the disease, while in reality they are simply the expression of slowly evolving degenerative changes in the cellular complexes of the basal ganglions engendered by a prolonged or even stealthily increasing inadequacy of the vascular supply. Finally, the occurrence of an abortive form of epidemic encephalitis is of the greatest importance and it is more than likely that the number of cases belonging under this head fully equals or even exceeds that of frankly developed cases. Here again the true origin of transitory or substantially recoverable though frequently severe types of psychoneurosis and mental disorder may remain totally unsuspected.

I have seen a total of about sixty cases in which the diagnosis of epidemic encephalitis could be postulated with relative certainty. A factor on which I have relied to a considerable extent is the presence of changes in the cerebrospinal fluid, more particularly a cell count varying from 10 to 200 per cubic millimeter associated with a definitely positive globulin reaction and with otherwise negative findings, i. e., a negative Wassermann reaction and absence of micro-organisms. Many observers have reported series of cases in which the spinal fluid was almost universally negative. Such has not been my experience and in the great majority of cases the fluid was definitely pathologic. I believe that the laboratory findings depend very largely on the stage of the disease and that the conditions are analogous to those encountered in poliomyelitis, namely, the frequent disappearance of 
definite changes in the spinal fluid after two or three weeks. Aside from the fact that there are mild or abortive cases with minimum modifications of the spinal fluid, there can be no doubt that many cases of epidemic encephalitis come under proper observation long after the truly initial phase of the disease. This combination of factors may account in part at least for the apparent discrepancies in the report of different clinicians.

Included in the series of sixty cases which have come under my observation were instances which correspond to most of the types thus far described in connection with this disease. Though pains in the extremities and cerebellar manifestations were not particularly uncommon, I have not seen frankly polyneuritic, herpetic or cerebellar types. I have seen, however, eight or nine cases which deserve to be designated as choreopsychotic and choreo-athetoid types of epidemic encephalitis because such manifestations represented in these cases the persistently predominating features of the clinical picture. Inasmuch as these clinical forms do not seem to have attracted much attention, at least so far as one may judge from the relatively meager reference to them in the literature, it has seemed useful to publish a few case histories which serve to illustrate these rather unusual types of epidemic encephalitis. This does not mean, of course, that either choreiform twitchings or psychotic manifestations have not been frequently recorded in connection with the disease. On the contrary, in the very earliest accounts of lethargic encephalitis emanating from Great Britain and France, more particularly in the report of the special British government board ${ }^{1}$ and in the initial communications of Netter, ${ }^{2}$ the occurrence of choreiform or analogous movements in some cases was emphasized. In this country, Bassoe ${ }^{3}$ was apparently the first to note these same phenomena which were present in four of the twelve cases originally reported by him. The choreiform twitchings involved the extremities and in two of the cases were confined to the left leg.

In all of these cases, however, the choreiform jerkings simply represented one of the many striking features in the clinical grouping. The same is true of another hyperkinetic phenomenon more recently identified and consisting of rapid rhythmic myoclonic twitchings in the abdominal wall, the face and the extremities, resembling the shocklike contractions induced by electrical stimulation. This myoclonus,

1. Report on an Inquiry Into an Obscure Disease, Encephalitis Lethargica, Local Government Board Reports on Public Health and Medical Subjects, N. S. 121, London, H. M. Stationery Office.

2. Netter: L'encéphalite léthargiéque, Paris méd. 8:8 (Aug. 3) 1918.

3. Bassoe, Peter: Epidemic Encephalitis (Nona), J. A. M. A. 72:971 (April 5) 1919. 
which was described more particularly by Sicard and Kudelski, ${ }^{4}$ Sabatini, ${ }^{5}$ and Reilly, ${ }^{8}$ is quite commonly observed, now that attention has been directed to it, in cases of epidemic encephalitis irrespective of the clinical type under observation. In some instances, however, it constitutes such a predominating feature that Sicard and Kudelski were led to isolate a special form to which they have given the name of "encéphalite aigüe myoclonique." In this type of the disease the myoclonic twitchings are described as brief, rapid and explosive, of distinctly rhythmic character and involving the musculature of the limbs, face and diaphragm; they are preceded by lancinating pains, headache and moderate fever and persist throughout the delirious and stuporous manifestations which eventually develop and usually prove fatal. The ordinary symptoms of epidemic encephalitis, such as somnolence and ocular palsies, are wanting. Sabatini likewise has laid great stress on the frequent occurrence of myoclonic phenomena and has given an excellent description of the symptom which fully tallies with that of the preceding authors. Sabatini insists on the fact that in some cases the jerkings simulated actual chorea. It should be stated, however, that there are very essential differences between chorea and rhythmic myoclonus, and that in reporting the present series of cases $I$ have in mind truly choreiform or choreo-athetoid manifestations and not regular rhythmic spasms.

As regards psychic disturbances in epidemic encephalitis, their common occurrence has been signalized from the very start and many authors have called attention more particularly to the nocturnal agitation with delirious states of hallucinatory or even delusional character. Here again in the majority of cases the psychic disorder has been merely one symptom of an otherwise well characterized instance of the usual type of epidemic encephalitis. In some cases, however, the mental confusion, agitation, hallucinosis, etc., have been so markedly predominant as to quite overshadow all the other features of the disease and to legitimate the description of an "acute psychotic type." Tilney ${ }^{7}$ has thus designated this form of the disease. Rather similar observations were published by House ${ }^{8}$ and others, and as far as

4. Sicard and Kudelski: L'encéphalite aigüe myoclonique, Bull. de la Soc. méd. d. hôp. 44:94 (Jan. 23) 1920.

5. Sabatini: Lethargic Encephalitis: Symptoms and Course, Policlinico 27:97 (Jan. 26) 1920; abstr., J. A. M. A. 74:1057 (April 10) 1920.

6. Reilly, Thomas F.: Hitherto Undescribed Sign in Diagnosis of Lethargic Encephalitis, J. A. M. A. 74:735 (Mrach 13) 1920.

7. Tilney: Epidemic Encephalitis, A Preliminary Consideration of Some of Its Prominent Clinical and Pathological Manifestations, Neurol. Bull. 2:106 (March) 1919.

8. House, W.: Epidemic (Lethargic) Encephalitis, J. A. M. A. 74:372 (Feb. 7) 1920. 
the psychic disturbances are concerned, the cases reported by these authors were remarkably similar to those described in the present artiele.

The combination of marked and persistent psychic disturbances with incessant choreiform jerkings as the outstanding features of a syndrome produced by epidemic encephalitis appears to be far more uncommon, and typical cases of this sort were not encountered in the literature until very recently when a further contribution to this subject by Bassoe ${ }^{9}$ came to my notice. Two of the cases reported by this author somewhat resemble those which have come under my observation and which are herein designated as the choreopsychotic type. This form of the disease is, I believe, far more common than is generally supposed.

Typical choreic and choreo-athetoid or frankly athetoid syndromes and other related spasmodic states occasioned by epidemic encephalitis have been particularly infrequent and have been encountered mainly as tardy or residual developments. Cases belonging under this heading have been reported by Pierre Marie and Mlle. G. Lévy, ${ }^{10}$ Souques, Lesne $^{11}$ and von Economo. ${ }^{12}$ Marie and Lévy published the case histories of seven out of thirteen cases of this kind. In two of the cases the hyperkinetic phenomena were essentially choreic in type, while in four others the movements consisted of rather coarse displacements of great amplitude in the leg or arm, or in both extremities on the same side, accompanied by oblique flexion or extension of the head. These spasmodic manifestations were not rapid and jerky but rather slow and relatively rhythmic and coordinate. The authors feel that they cannot assimilate these disorders to any of the actually recognized categories of involuntary spasmodic phenomena. Lesnés article deals essentially with the development of choreiform movements after the acute stage of lethargic encephalitis or as a sequel of influenza. Personal observations are recorded and the earlier experiences of Netter, Marie and Souques with analogous conditions are summarized. Von Economo has reported an interesting case of lethargic encephalitis remarkable for its chronicity, in which the condition

9. Bassoe, Peter: The Delirious and the Meningoradicular Types of Epidemic Encephalitis, J. A. M. A. 74:1009 (Apri1 10) 1920.

10. Marie, Pierre, and Lévy, Mlle. G.: Plusieurs cas de mouvements involontaires d'aspect particulier apparus après un épisode fébrile grippal et, pour certains, après des signes d'encéphalite léthargique, Rev. neurol. 35:300, 511, 1919.

11. Lesné: Mouvements choréiformes observés à la suite de l'encéphalite léthargique ou de l'influenza, Bull. Soc. méd. d. hôp., Nov. 29, 1918.

12. Von Economo: Encephalitis lethargica chronica, München. med. Wchnschr. 66:1311 (Nov. 14) 1919. 
had an acute onset and exhibited a number of remissions until more serious involvements eventually. appeared and proved fatal almost two years after the initial symptoms. The patient gradually developed the syndrome of pseudobulbar paralysis associated with typical athetoid manifestations. It was characterized by dysphagia, dysarthria, dysphonia, paresis of the lingual and palatal musculature, generalized rigidity, spasmodic laughter and choreo-athetoid movements in the face as well as in the upper and lower extremities. The transitory improvement concerned more especially the cranial nerve functions whereas the athetoid disorders steadily increased in severity. At times, however, all the symptoms seemed to recede only to return in more alarming form.

\section{CHOREO-ATHETOID TYPE}

CASE 1.-C. W., aged 57, married, a traveling salesman, was seen by me May 5, 1919, about three months after the onset of his disturbances. Reliable data were obtained from his family physician regarding the initial phase of his disease.

History.-His family and personal history yielded nothing of significance for the interpretation of his actual nervous disorder. Both the patient and his physician were quite sure that he had not had influenza and that the first episode in his illness was the appearance of a large and annoying carbuncle on the back of the neck during the first days of February, 1919. Shortly thereafter the patient became feverish and drowsy, complained of blurred vision and diplopia and developed bilateral facial paralysis. There was neither vertigo nor vomiting, but pain of considerable severity was felt over a circumscribed area in the right parietal region which, judging from the patient's own description, had all the characters of "clavus hystericus." He soon showed evidence of some weakness in the extremities on the left side and exhibited restless movemients in the trunk and extremities. He was seen at that time by a consultant and lumbar puncture was performed. The spinal fluid is said to have been entirely negative. The blood and urine were likewise negative. The patient had a slow and tedious convalescence with unsatisfactory retrogression of some of his disturbances and decided aggravation of others.

Examination.-When seen by me for the first time, May 5, 1919, he complained of recurrent facial weakness, disordered speech and uncontrollable movements of his face and extremities. Neurologic examination showed that his contentions were justified. Whether standing or sitting some part of the body was almost constantly in motion, the face and left lower extremity assuming the major share of the otherwise general instability. In the sitting posture the trunk exhibited rather slight oscillations either forward and backward or laterally, the head was slowly rotated from side to side and at the same time thrown slightly backward, the eyes were held semiclosed by the constant wrinkling of the forehead and orbiculopalpebral spasm, the expression being typically that of one attempting to distinguish a distant object in the full glare of the sun, with perhaps the added appearance of acute anxiety and distress. The angle of the mouth on either side was likewise occasionally retracted and the lips curled or twisted in various ways. The hands and forearms were now and then the seat of restless fidgety movements of prona- 
tion together with clutching movements of the fingers, but the unrest manifested in the upper extremities was minimal when compared with that observed in the lower extremities. There was ceaseless shuffling of both feet and more particularly of the left foot which was constantly displaced forward and backward or laterally, or else only the heel or forepart of the foot was alternately moved inward and outward. Occasionally the knee was repeatedly adducted and abducted. His gait showed slight dragging of the left lower extremity with uneven stepping. There was no gross paralysis in the limbs, he could execute all the ordinary movements usually tested but his motor power was generally subnormal with more appreciable loss on the left side. His grasp, registered with Mathieu's dynamometer, gave 70 on the right side and $50 \mathrm{on}$ the left. The tendon reflexes were all hyperactive and distinctly more so on the left side, but neither ankle clonus, Babinski nor Oppenheim phenomena could be elicited. The abdominal and cremasteric reflexes were not obtained. The left facial nerve was decidedly paretic in its lower branch, faintly so in its upper branch. Ocular excursion was excellent in all directions save as regards convergence which was practically nil. The pupils were small, perfectly rounded and reacted neither to light nor to accommodation. The eyegrounds were negative. His speech was dysarthric with occasional approach to explosiveness and his voice was distinctly high pitched. The tongue was not deviated when first protruded though it was afterward constantly shifted from side to side. The soft palate and pharynx appeared functionally normal. During the entire consultation the patient emitted plaintive grunting sounds, which manifestations may, however, have been purely hysterical features as the man had always been a rather high strung nervous individual. This symptom was not present at subsequent examinations. There were no disturbances of superficial and deep sensibility nor of the special senses and the general physical findings were negative. The intellectual faculties were absolutely intact.

Course.-I did not have the opportunity of seeing this patient again until Sept. 23, 1919. He then complained of transitory pain and soreness on the top of the head and was still restless, uneasy and mentally distressed by his prolonged illness. Physical examination revealed, however, a striking amelioration. His motor strength had largely returned, his grasp now registered 120 and 110 with the dynamometer, his facial palsy had entirely cleared up and his gait was normal. The pupils, however, remained totally reactionless, speech was still defective with a tendency to explosive features and he continued to exhibit the slow unceasing contortions of the face and the restless movements of the left leg and foot. The grimaces and head displacements had all the characters of genuine athetosis whereas the movements in the extremities were more closely akin to choreiform disturbances.

He was seen again Nov. 12, 1919, Jan. 12, and April 16, 1920, and his condition had not materially changed except as regards steady improvement in general strength and nutrition.

CASE 2.-L. S., a man 27 years of age, married, employed in a cigar factory, born in Poland, which he left when still young, could give no definite information concerning his family history.

History.-Aside from the ordinary diseases of childhood, he had always enjoyed excellent health, was a diligent worker, used tobacco in moderation and denied alcoholic indulgence and venereal infection. He was sent to me, Oct. 10,1919, by a former pupil for an opinion regarding the nature and prognosis of very severe muscular twitchings which had developed progres- 
sively after an obscure meningeal disorder associated with severe mental derangement. The history of the events which led up to his present condition was supplied by his physician who stated that the patient had had a severe attack of influenza during November, 1918, from which he recovered with extreme slowness. Early in January, 1919, he rapidly developed acute mental disturbances with maniacal agitation and during three weeks was absolutely out of his mind and had to be constantly watched and frequently restrained. A detailed account of all the features of this illness unfortunately could not be obtained except the fact that this transitory mental disturbance was accompanied throughout by fever, rigidity of the neck and frequent jerky movements in the head and extremities. Meningitis was suspected and lumbar puncture performed. The fluid was clear, contained many cells and gave a strongly positive globulin reaction but contained no organisms. The Wassermann reaction was negative. He improved after the lumbar puncture and his psychic manifestations rapidly subsided but in the latter part of February, about three weeks after the cessation of his febrile and delirious state, he began to develop almost constant choreiform movements which became practically generalized.

Examination.-Neurologic examination, Oct. 1, 1919, disclosed as the most striking and prominent feature decided spasmodic excursions of the patient's head consisting of constant, rather coarse and ample displacements, somewhat resembling those of Huntington's chorea though far less rapid in execution. The head was rotated from side to side and especially obliquely hyperflexed on the chest then obliquely hyperextended backward in such a way that during the extensor phase the ear was approximated to the shoulder and the face turned upward toward the opposite side, thus recalling the appearance seen in spasmodic torticollis. At the same time the face exhibited the most diverse contortions, such as slow winking and frowning movements, retraction of the corners of the mouth or puckering of the lips, occasionally interrupted by more rapid myoclonic twitchings of the entire musculature of the face, particularly on the right side. The right arm and leg likewise exhibited almost constant movements, but these were relatively less striking, were almost confined to the distal segments of the extremities and consisted of pronation and supination of the forearm and hand, flexion and extension at the wrist, flexion and extension as well as abduction of the fingers, adduction and elevation of the foot and flexion and extension of the toes. There were no gross movements at the larger joints of the extremities but occasional shrugging of the shoulders and irregular oscillations of the trunk were visible when the patient changed from the recumbent to the sitting posture or from the latter to the standing position.

There was no Romberg sign and the gait presented nothing unusual though the patient maintained that he frequently could not control his steps, felt inclined to pitch forward and sometimes walked like a drunken man. As usually occurs in ordinary chorea the involuntary movements in the extremities stopped completely or almost when the patient executed commands, and members of his family stated that all twitchings usually ceased during sleep. It was difficult to designate correctly the spasmodic movements observed in this patient; they had nothing in common with the rhythmic myoclonic manifestations seen in various parts of the body in a fair percentage of cases of epidemic encephalitis; they borrowed the features of both chorea and athetosis and for this reason it has seemed more practical to label this form the choreo-athetoid type. In reality, however, confusion existed mainly as 
regards the twitchings in the extremities which greatly resembled choreiform movements though they were much less rapid and jerky than the latter and recalled by their relative slowness and labored execution the usual appearance of athetoid spasms. The spasmodic phenomena exhibited by the head and face were, on the contrary, absolutely identical with those seen in typical cases of bilateral and hemi-athetosis.

There was no motor paralysis in the extremities, but a certain element of rigidity was demonstrable more especially on the right side. With Mathieu's dynamometer the grasp was 120 on the right and 100 on the left. The tendon reflexes were very active but of symmetric intensity and were not accompanied by clonus nor by Babinski and Oppenheim phenomena. The tongue was protruded in a jerky manner and rolled from side to side or curled up in the roof of the mouth. The soft palate and pharynx appeared normal and phonation was perfect but speech was somewhat defective with frequent approach to stuttering or explosive.in character, perhaps by reason of the unceasing labial spasm. A definite palsy of the right lower facial nerve was evident. Ocular excursion, pupillary responses and eyegrounds were normal. The special senses, general sensibility and sphincter control were undisturbed. The patient was anxious regarding the outcome of his affection and distressed by his enforced inoccupation but presented no evidence of mental deterioration. His mode of behavior, attention, memory, and judgment were all carefully studied and there appeared no residue of his previous psychic derangement. The heart, blood pressure, blood and urine examinations yielded negative findings. The patient had come from a distant city and unfortunately was never seen again despite repeated endeavors to locate him.

CASE 3.-F. R., a married housewife, 34 years of age, had had no children and no miscarriages, and aside from a rather prolonged attack of chorea at the age of 14 and recurrent tonsillitis her past history presented nothing of unusual interest.

History.-During the latter part of February, 1919, she had a moderately severe influenzal infection from which it was claimed she never fully recovered. Early in June, 1919, she began to have pains in the back, neck and shoulders, felt weak and unsteady on her feet, complained of not seeing objects clearly, seeing double whenever she directed her gaze to the right and having distaste for all food. Within a few days she developed slight fever, muscular twitchings in the neck, hands and left hypochondrium and became so drowsy that her family physician became alarmed and requested a consultation.

Examination.-When first seen by me, June 10, 1919, the patient lay perfectly quiet in bed apparently in a peaceful slumber with flushed face, moist skin, rather slow and irregular respiration and a temperature of $101.2 \mathrm{~F}$. She could be aroused without difficulty, executed commands quite well and answered all questions intelligently. She stated that she no longer had pains anywhere except in the back of the head but felt terribly tired and saw everything through a heavy veil or mist. She remained perfectly immobile during questioning except for occasional rather rhythmic and shocklike twitchings in various parts of the body, notably in the neck, left arm and abdominal wall. The cervical spasm tilted the head backward and to the left; in the arm the main movement occurred at the wrist and consisted of spasmodic flexion of the hand resembling cortical spasm, while in the abdomen there was rhythmic recession of the left hypochondrium somewhat analogous to what takes place in hiccough. Rarely, similar though less typically myoclonic twitchings were likewise seen in the left leg or on the right side of the 
body. These various spasms were rapid and jerky like those of chorea but differed from choreiform manifestations by reason of their relative uniformity or rhythmic character. There was a faint drooping of the eyelids and a distinct though asymmetric bilateral prosopoplegia, the right facial musculature being more involved than the left. The physiognomy presented a fairly typical Parkinsonian mask perhaps conditioned by the bilateral facial weakness. The eyes followed the finger in all directions but excursion was slow and attended by definite nystagmus, both horizontal and vertical. The pupils reacted sluggishly and imperfectly to light and to convergence. The eyegrounds were distinctly congested and the disk outlines hazy. There was slight dysarthria but no involvement of the tongue, soft palate or pharynx could be detected. The extremities were free from actual paralysis but a tendency to generalized rigidity was present and the tendon reflexes throughout were hyperactive and equal on the two sides. There was neither Babinski nor Oppenheim phenomenon. The arms presented no evidence of either ataxia or adiadokocinesis but an intention tremor appeared when the patient carried the finger tip to the nose or attempted to take a small object from the examiner's hand. General sensibility, special senses and.organic functions were undisturbed. Owing to the gravity of the patient's illness it seemed unwise to get her out of bed for the purpose of testing her gait, but it was learned from the family that for two or three days prior to taking to her bed the patient walked in a very irregular fashion, her body swaying from one side to the other. Lumbar puncture was advised but refused. Three days later the patient's condition was decidedly worse, the drowsiness greater, the fever higher, the twitchings more accentuated and constant and furthermore nocturnal agitation with hallucinatory manifestations appeared. It was then decided to proceed with lumbar puncture. The fluid came out under considerable tension, was perfectly clear, contained 56 cells to the cubic millimeter, gave a strongly positive globulin reaction, a negative Wassermann reaction and an indefinite gold curve. The blood showed a leukocytosis of 17,000 and yielded a negative Wassermann reaction. The patient's condition seemed to have been materially improved in every respect, but the involuntary spasmodic phenomena persisted. A second lumbar puncture, performed June 20, 1919, showed a cell count of 35 and a still markedly positive globulin reaction.

Course.-From this time on steady amelioration occurred and the further history was supplied by the physician in charge who stated that within a week the temperature became normal and remained so. The drowsiness, ptosis, nystagmus and dysarthria disappeared within three weeks and by that time the patient had been allowed to be up and about the house. She continued, however, to present her peculiar jerkings of the head and the restless movements of the hand which had rather increased. Her convalescence was completed in the country and I unfortunately did not see the patient again until several months later.

The patient finally reported at the office, Dec. 15, 1919, in a state of profound mental depression because of the uncontrollable twitchings which practically incapacitated her and rendered life intolerable. To my great surprise her spasmodic manifestations had not only very decidedly increased in severity but had moreover assumed quite a different character. The musculature of the neck and shoulders was the seat of a most extraordinary and persistent spasm. The patient's head was rigidly hyperextended, then slowly rotated from side to side and at the same time deeply buried between the 
spasmodically elevated shoulders, giving the impression of an attempt at telescoping the head through the trunk. The spasm, which was essentially tonic in character, lasted two or three minutes, evanesced, then reappeared after an interval of from a few seconds to a minute and was accompanied during the greater part of its exhibition by tonic bilateral contraction of the platysma myoides, more marked on the right side. Had I not seen this patient during the acute phase of her malady, I would have been much inclined to look on this cervical spasm as a purely hysterical phenomenon. However, the other twitchings also had persisted and were plainly in evidence. During the spasmodic excursions of the head, which was more commonly lowered toward the left shoulder with consequent deviation of the chin toward the right, the facial musculature likewise participated in the elaboration of the twitching phenomenon, being animated by irregular pouting of the lips and frowning movements in the forehead. The left upper extremity, particularly the hand and fingers, constantly executed slow and irregular movements closely resembling athetoid spasm. The hand was pronated and deviated outward or hyperextended and the fingers alternately flexed and extended or spread apart, the thumb and index finger being apparently more involved than the other fingers. Only an occasional restless displacement was observed in the left foot. No contractions occurred at the larger joints of the extremities on the left side and none was seen anywhere on the right side of the body save the spasmodic shrugging of the shoulder previously noted. There was no evidence of motor weakness although an accurate evaluation of the grasp of the left hand was rendered impossible by the constant displacements of the fingers. The whole left side seemed distinctly more rigid than the right and exhibited more active tendon reflexes. There was neither ankle clonus, Babinski nor Oppenheim phenomenon. The patient was a highly nervous woman and developed a marked tremor throughout the upper extremities when asked to hold the arms quietly extended in front of her with fingers spread widely apart. A trace of ataxia was present in both arms. Ocular excursion was perfect in all directions and the pupils were rather small though yielding normal light and accommodation responses. The eyegrounds still showed rather full and tortuous vessels with otherwise negative disks. Examination of all of the other cranial nerves was negative save for the various muscle spasms previously mentioned. General sensibility, sphincteric control and trophic functions were intact. The heart dulness could not be satisfactorily mapped out owing to the patient's adiposity, but a loud systolic murmur was present over the apex and distinctly transmitted toward the scapula.

The patient was seen again Jan. 5, Feb. 26, and April 14, 1920, and while she thought that some improvement had taken place under administration of bromids and hyoscin hydrobromid, I could see no material change in her various spasmodic manifestations.

\section{SUMMARY OF CHOREO-ATHETOID SYNDROME}

All three cases included in the preceding series developed, within a period of three months after a typical attack of epidemic encephalitis, a syndrome characterized by choreo-athetoid spasms in the face, neck and extremities. The original manifestations of the encephalitis corresponded to the usual clinical type except in one case in which very severe mental disorder and maniacal agitation constituted the essential 
features of the symptomatology. None of the patients presented at any time evidence of external ophthalmoplegia, but in one case a permanent paralysis of the intrinsic ocular musculature was noted. In only one case was definite lethargy observed while in all three the facial musculature was distinctly involved either on one or on both sides. In a general way the so-called characteristic triad, lethargy, asthenia and oculomotor paralysis was reduced to its minimum expression in this series of cases. The predominating feature in all was the rapid and progressive elaboration of a choreo-athetoid syndrome essentially unilateral in distribution and remarkable for the exaggerated participation of the cervical and facial musculature. It is interesting that in all three cases more or less definite hyperkinetic phenomena of this same type were already recognizable in the initial phase of the disease. While the character of the twitchings at first was perhaps not absolutely pathognomonic, the spasmodic movements in the later or convalescent stage of the affection were fairly characteristic, being of the nature of athetosis in the face and neck and on the contrary more closely analogous to choreiform jerkings in the extremities. In Case 3 , however, the spasms eventually assumed almost classic athetoid features, both in the neck and in the extremities.

\section{CHOREOPSYCHOTIC TYPE}

CASE 4.-G. A., a married housewife, aged 38 , was seen by me only once and her history was obtained from the attending physician on the occasion of a consultation held Oct. 17, 1919.

History.-Aside from what was regarded as a relatively mild and even doubtful attack of influenza in the early part of September, 1919, there was nothing of unusual importance in her history. The first symptoms of her illness had appeared from eight to ten days before I saw her and consisted mainly of severe pains in the left occipital region, in the back of the neck and left shoulder and radiating into the left arm. This was interpreted at first simply as neuralgia. Within two or three days, however, it was followed by mild fever, diplopia, peculiar nodding movements of the head, muscular twitchings in the left arm and rapidly increasing tendency to nocturnal agitation, mental confusion with marked disorientation and eventually by hallucinatory delirium. She was fairly quiet during the day although at no time lethargic and rarely slept more than an hour at a time. The physician and the two attending nurses stated that at night more especially the patient constantly tried to get out of bed, had to be closely watched, fretted about the care of her house and spoke of nothing except the necessity of personally directing the management of her household. She would sit up in bed, point her finger toward a corner of the room and, as though speaking to her servant whom she called by name, she would carry on an imaginary conversation, giving detailed orders for tasks which it was known she had previously planned to carry out. She would insist on definite answers and accounts, remonstrated, pleaded or approved, and continued in this manner a good part of the night. She was totally disoriented for time and place 
but recognized her doctor every time he came into the room and named him correctly. During the day she had lucid intervals when she seemed to realize that her mind had wandered the greater part of the time and she would herself call attention to the silly things she had said and done, but usually she would almost immediately lapse back into her dreamy and confused mental state. Her temperature had oscillated between 99.6 and 100, and never exceeded $101.6 \mathrm{~F}$. She took nourishment rather reluctantly, had no difficulty in swallowing and retained control of the sphincters.

Examination.-When I saw the patient Oct. 17, 1919, she was lying on her back with half closed eyes and a dreamy countenance. The first fact which attracted my attention was the peculiar and practically constant nodding of the head. This consisted in a fairly rhythmic and rather jerky spasmodic rotation of the head to the right with very slight associated backward tilting. The left arm also exhibited constant irregular and jerky movements consisting of pronation and supination of the forearm and hand, flexion and extension of individual fingers with frequent picking at the bed clothes. Restless movements and displacements also occurred in other parts of the body but were much less frequent and noticeable. By talking rather sharply to the patient and constantly urging her it was possible to proceed with a fairly satisfactory neurologic examination. At rest there was slight drooping of both eyelids and an appearance of mild divergence of the eyeballs but the patient could be made to follow the finger in all directions. No definite ophthalmoplegia was evident but a well marked vertical nystagmus was present and the patient claimed that she saw double whatever the direction of her gaze. The pupils were small and reacted sluggishly to light. The eyegrounds were distinctly congested and the outline of the right disk poorly defined. The facial musculature was flaccid on both sides and showed appreciable paresis. There was no gross paralysis in the extremities and all movements were executed but the left arm appeared diffusely paretic and atonic. Both arms presented slight ataxia and well marked intention tremor. When the patient sat up in bed the nodding of the head became less constant and was largely replaced by a coarse intention tremor such as occurs in advanced cases of multiple sclerosis. The tendon reflexes were all hyperactive but without accompanying ankle clonus or Babinski-Oppenheim phenomena. The abdominal reflexes were abolished and the stroking intended to elicit them was followed by unusually pronounced dermographia. The tongue showed no deviation but was only imperfectly protruded and speech was slow, monotonous and definitely dysarthric though usually intelligible. The temperature at this time was $99.8 \mathrm{~F}$, the pulse rate between 110 and 120 and the respirations rather shallow and irregular. Lumbar puncture yielded a perfectly clear fluid under moderately increased pressure. The cell count per cubic millimeter was 32 , the globulin reaction strongly positive $(+++)$, the Wassermann reaction negative in all dilutions and the colloidal gold curve negative.

Course.-Four days later I was informed by the attending physician that lumbar puncture produced no amelioration in the patient's condition. She continued her nocturnal agitation and hallucinatory manifestations but rapidly became more drowsy during the day, showed signs of increasing exhaustion, developed dysphagia and hypoglossal paralysis and died three days after I saw her from respiratory paralysis. Unfortunately a necropsy could not be secured. 
CASE 5.-J. W., aged 43, a man, married, a shipping clerk by occupation, was seen in consultation with his physician, Jan. 30, 1920.

History.-Aside from an attack of sunstroke several years before, his past history was exceptionally good and he had always been a perfectly healthy and active man. No influenzat infection preceded the present illness which began, January 24, with paroxysms of pain in the occipital-region and diplopia. The latter symptom annoyed him so much that he had all the pictures on the wall of his room removed. His temperature rose to $101 \mathrm{~F}$., and remained at that point for a few days; the diplopia disappeared after three days, but the occipital pains persisted and at times gave him considerable distress. There was no paralysis anywhere at this time according to his physician and the superficial and deep reflexes were undisturbed. The patient soon assumed a drowsy and passive attitude and would lie for hours with eyes closed, totally indifferent to whatever took place about him and except for slight choreiform twitchings in the upper and lower extremities appeared perfectly quiet as though in a peaceful slumber. He could be aroused without difficulty, carried on conversation regarding the weather conditions, his state of health, etc., but soon drifted into details pertaining to the work in which he was engaged prior to the onset of his illness. His work seemed uppermost in his mind and he frequently muttered something in regard to it, became markedly disoriented for both time and place, but recognized those about him and called them by name though he took no further interest in their doings and never passed any remarks when letters received from a member of his family who had just left for a neighboring city were read in front of him. At night especially, though sometimes during the day, he became more markedly confused and restless, would sit up in bed and talk incoherently for long periods, almost invariably referring to some phase or other of his occupation and exhibiting by both word and gesture unmistakable evidence of hallucinatory delirium. The necessity of carrying out some given task seemed all absorbing and he frequently insisted on getting hold of a box he had to fix. He tried to get out of bed on several occasions but was never violent and could always be induced to lie quietly and obey the doctor's orders.

Examination. - When I first examined this man, January 30 , I was surprised to find relatively little objective evidence of serious organic disease. He was rather somnolent but easily aroused, answered questions quite well, executed all commands and appeared perfectly rational. He readily lapsed back, however, into his habitual state of indifference and mental inertia. Restless and jerky movements were observed in the extremities but only at intervals and consisted in the arm of flexion at the elbow, flexion at the wrist and ulnar deviation of the extended and abducted fingers. In the lower extremity abduction of the thigh and extension of the foot and toes predominated though at times the whole limb was the seat of a more violent kicking movement. These various twitchings had a jerky spasmodic character and involved all four extremities but were rather more frequent and pronounced on the right side. No analogous twitchings were seen in either the face or neck. A very faint hemiparesis was present on the right side affecting the lower facial region as well as the extremities. Otherwise no paralytic disturbances were observed. All of the cranial nerves were intact and not the slightest implication of the ocular muscles could be detected. The pupils.were small, in fact almost myotic, but nevertheless yielded normal light and accommodation reflexes. The cutaneous reflexes were rather sluggish 
but the tendon jerks were readily elicited though without appreciable exaggeration. The plantar reflexes were normal. No disorders of general or special sensibility were discovered and sphincter control was perfect except during a period of twenty-four hours at the beginning of the second week of the illness when the patient had to be catheterized. Examination of the eyegrounds disclosed negative findings.

Course.-This was the mildest case of the series and the entire symptomatology consisted at this time of more or less drowsiness, fairly persistent mental confusion, disorientation with episodic hallucinosis and jerky twitchings in the extremities. Lumbar puncture gave a perfectly clear spinal fluid with a direct cell count of only 15 per cubic millimeter but a strongly positive globulin reaction $(+++)$. The Wassermann and colloidal gold reactions were negative. Unfortunately no blood examination was made. The urine showed no abnormal constituents. The patient was apparently markedly benefited by lumbar puncture and was much clearer mentally and quieter physically for three or four days, after which the former state of affairs gradually reappeared. He was seen again, February 13, and seemed to me much less drowsy than on the previous occasion and less restless and jerky. A second lumbar puncture was performed, the cell count was reduced to 7 and the globulin reaction was only faintly positive $(+)$. From this time on the patient showed rather steady improvement in all respects and during the fifth week began to sleep in more natural fashion and at more conventional hours. The pseudochoreiform twitchings gradually subsided and he took a more active interest in his family and surroundings. Nothing further of importance was recorded by his physician. The patient at my request reported at the office for examination, April 22, and a complete survey of the neurologic functions was carefully made. He complained of paroxysmal twinges of pain in the right suboccipital area and right side of the face but apart from infrequent winking of the eyes and very defective wrinkling of the forehead and elevation of the upper lip, all of which gave him a rather definite Parkinsonian facial appearance, absolutely no objective evidence of residual disturbance was detected. No twitchings were observed anywhere and his psychic faculties were intact. He was considered to have virtually recovered.

CASE 6.-T. G., a man, 45 years of age, married, assistant manager of a contracting and building concern, an Italian by birth, a rather high strung and impulsive individual, was considered an unusually able business man, used tobacco rather excessively but aside from light wine at occasional dinners never indulged in alcoholic stimulants.

History.-With the exception of the ordinary diseases of childhood and typhoid fever at 20 , he had always enjoyed remarkably good health and was steadily active at his work. In the early part of December, 1919, he contracted a severe cold and subsequently developed influenzal manifestations with fairly sustained high temperature and apparently bilateral bronchopneumonia. He was confined to his bed for about three weeks and took fully another month to regain his strength and energy. He came home, Feb. 20,1920, complaining of lassitude, headache, dizziness, pains in the back and legs and blurred vision. His family physitian was called, found no fever but nevertheless ordered him to bed and gave him a calomel and saline purge together with aspirin. The following evening he felt much better and planned to return to his office on the succeeding morning but not being permitted to do so became greatly exercised and agitated, abused the doctor, refused to eat and quarreled with every one in the house. From this time on he rapidly 
developed quite a striking series of motor and psychic disturbances, became restless, paced the floor a large part of the time, showed tremor in the hands and irregular facial twitchings, would not go to bed and constantly talked of gigantic building, plans which would place him at the head of the largest building corporation in the country. During the night he would occasionally lie down for an hour or so but even then continually muttered to himself or called out in a loud voice, giving orders to his subordinates and carrying on lengthy conversations with his associates. At no time did he show any evidence of drowsiness or apathy.

When I saw the patient in consultation, March 2, 1920, he was still restless but perfectly manageable and even morbidly good-humored though he became antagonistic the moment he learned the purpose of our visit. He persisted in saying that he did not need the services of a physician, that he was not ill, had never felt better, in fact, but that nobody understood him, that his own people were against him, believing him mentally unsound when in reality he was conceiving great schemes which were bound to triple the business of his firm and lead to his own advancement. He confided to the doctor that he had been most fortunate in recent speculations, had large sums stored away in some New York banks and that he contemplated building a magnificent residence in the suburbs of the great metropolis where his wife and two daughters would finally have the opportunity of entering the world of wealth and culture to which they belonged. This and similar grandiose ideas were the dominating features of his mental operations and evidently accounted for his visible exuberance and the outbursts of laughter which frequently interrupted our subsequent examination. During most of this introductory conversation he either stood up, shuffling his feet, or paced the floor; occasionally he sat down, but some part of the body was constantly in motion and one was instinctively reminded of what Charcot called "choreiform instability." One foot or the other was pushed forward or retracted, the heel or the forepart of the foot was displaced mesially or laterally, the knee was repeatedly adducted and abducted, the hand and forearm were slightly pronated and supinated, the hand was extended and irregular drumming or grasping movements of the fingers were executed. These various movements had a jerky and spasmodic character, and occurred on both sides of the body but were more pronounced on the right side. The head was frequently turned to one side, but without spasmodic element, and facial twitchings consisting especially of a clonic quiver of the cheeks and elevation of the eyebrows were almost constant. The facial contractions were much accentuated when he was requested to follow the finger with the eyes, close the eyes or elevate the upper lip. The twitchings in the extremities on the contrary diminished when the patient carried out designated movements and were replaced by a coarse tremor.

Examination.-He was a rather thin but muscularly developed individual of medium stature. The various tests for motor functions revealed no paral$y$ sis in the extremities but a marked and rather coarse intention tremor appeared whenever he held the extended arms directly in front of him or carried the finger to the tip of the nose. A similar tremor was present in either lower extremity on elevating it to touch the examiner's hand with the big toe. The tremor did not progressively augment in amplitude as is usual in multiple sclerosis, and no associated ataxia or dysmetria was observed. His grasp was powerful and equal on the two sides. The tendon jerks were all hyperactive but unaccompanied by either clonus or pathologic responses 
of the great toe. The umbilical and cremasteric reflexes were retained. The cranial nerves presented only slight abnormalities. There was a definite ptosis on the left side and the facial musculature on that side was relaxed, the angle of the mouth drooping distinctly on elevation of the lip. Ocular excursion was excellent in all directions save for convergence which was practically nil, and a faint nystagmus appeared when the eyes were carried to either the extreme right or left. The pupils were small, perfectly rounded, equal in size and reacted neither to light nor to accommodation efforts. Examination of the eyegrounds was negative. The tongue was protruded well and without deviation, but showed a fine fibrillary tremor at the tip and along the lateral borders. The closed eyelids presented a marked tremor. Speech was not materially affected, but occasionally the patient seemed unable to find his word, stuttered and mispronounced the word particularly if asked to repeat a difficult one. There was no involvement of the organic sphincters and no disorders of general or special sensibility.

In many ways the physical examination was unsatisfactory and many tests could not be properly carried out owing to the patient's psychic condition. His attention was easily diverted and it was plain that his mind was elsewhere, deeply engaged in the pursuit of his delusional consolations. Moroever, he constantly interjected sarcastic remarks regarding the examination, cracked jokes about doctors in general, or indulged in loud and prolonged laughter. This was clearly akin to what has been described as "witzelsucht" or "moria." His memory was shown by careful control to be grossly defective and he gave a most erroneous account of certain important incidents which had interested him prior to his influenzal infection.

Evidently the clinical physiognomy of this case was so strongly suggestive of general paresis that diagnosis was impossible without further observation and laboratory tests. Lumbar puncture yielded a perfectly clear fluid under moderate tension giving a cell count of 34 per cubic millimeter and a well marked butyric acid reaction for globulin $(++)$. The colloidal gold curve in this case showed a definite discoloration in the first four or five tubes, but the Wassermann reaction was negative in all dilutions. The blood Wassermann reaction was likewise negative. The leukocyte count was 11,000 and the urine practically normal in every respect.

Course.-The patient was rather more quiet, both physically and mentally, during the two days following lumbar puncture, but soon he began to exhibit the same motor unrest, irregular jerky movements and delusional manifestations of grandiose type. His various symptoms did not materially change until approximately two weeks later, when he suddenly became semicomatose after a night of unusual agitation with prolonged shouting and wild delirium. When seen on this occasion, March 18 , he was profoundly somnolent, could barely be aroused, merely muttered unintelligibly in answer to questions, would not protrude the tongue nor squeeze the hand, but nevertheless swallowed food placed in his mouth. His breathing was rapid and irregular, occasionally slower and stertorous, his pulse was rapid, oscillating between 120 and 130 , but perfectly regular, and his temperature by axilla $100.2 \mathrm{~F}$. This time he again almost constantly presented rapid and irregular jerky displacements of his extremities and facial twitchings. This stuporous state lasted a little more than a day and was followed by a recrudescence of the hallucinatory delirium and delusional wanderings. He was totally disoriented, no longer seemed to recognize those about him, refused medicine and sometimes food, talked incoherently the greater part of the time and lost all concept of propriety, voiding 
in bed or on the floor. A similar stuporous attack appeared March 24 and lasted almost three days. Lumbar puncture performed at this time again gave a perfectly clear fluid with a cell count of 60 and a strongly positive globulin reaction. Although considerable fluid was withdrawn, the procedure did not modify the patient's condition in the least and when he regained consciousness he at once started to display his previous motor agitation, mutterings and absurd ideas. He was, moreover, perceptibly weaker, seemed to use the right extremities in a weak and ataxic fashion and could be more easily restrained. He took little nourishment, swallowed with some difficulty and in a few days gradually became comatose for the third time, developed Cheyne-Stokes' breathing, paroxysmal hiccough, marked dysphagia and a rising temperature. The spasmodic jerkings eventually subsided and he died, April 4, after three days of coma.

Necropsy.-A limited necropsy was obtained, examination of the brain only being permitted, and we had to be content with removal of fragments for microscopic examination. However, enough information was secured to establish positively the anatomic diagnosis.

The brain was remarkably edematous both on the surface and on section and the leptomeningeal vessels at the base and over the frontoparietal region were distinctly engorged. The histologic findings corresponded to the lesions thus far described by other observers. There was only discrete infiltration of the pia with mononuclear cells, whereas the intracerebral vessels, more particularly the veins, were markedly distended and showed extensive adventitial infiltration. A diffuse interstitial infiltration was likewise present, but no definite glia hyperplasia was detected. Minute hemorrhages were found in the midbrain and corpus striatum, but were particularly abundant in the subcortex of the left frontal lobe. There were no advanced degenerative changes in the ganglion cells, but merely rarefaction with imprecision of the tigroid substance and a more or less diffuse staining reaction. These various lesions were practically identical at all levels examined, but seemed more severe and extensive in the cortex and particularly in the subcortex of the frontal regions. It was in this area that the vascular changes were most striking and the pial infiltration most pronounced. The larger pyramidal cells were largely deprived of chromatin granules and many were surrounded by the infiltrating cells. Nowhere did the blood vessels exhibit the characteristic structural modifications found in syphilis and paresis.

CASE 7.-I. W., a man, 40 years of age, married, a tailor by occupation, was admitted to the public wards of the Albany City Hospital, March 29, 1920, complaining of nervousness, weakness and pains in the neck, back and in both arms from the elbows to the tips of the fingers. These pains had occurred in paroxysms since a severe cold contracted March 8, 1920.

History.-His family and past history were unimportant. $\mathrm{He}$ stated that he had enjoyed relatively good health, had always been a hard worker, slept little, used alcohol in moderation and had never contracted any venereal infection.

The present illness began about March 8, 1920, when he contracted a severe cold lasting several days, during which he felt feverish and fagged out. Then paroxysmal intense pains appeared in the neck, back, chest and upper extremities. At the time of admission he stated that the pains were no longer of great severity and that numbness and tingling in the arms and hands were the most annoying symptoms. General physical examination by the house physician 
revealed nothing of serious nature and his temperature was normal, but he appeared nervous and restless and exhibited occasional facial twitchings and jerky movements in the extremities. Two days later he began to show evidence of mental confusion and disorientation, especially at night, and developed involuntary and incoordinate muscular twitchings throughout the body, but more particularly in the facial and abdominal musculature. His skin was unduly moist, his face pallid and his pulse rapid, ranging from 100 to 130 . His symptoms rapidly became more accentuated and mental confusion, marked disorientation for time and place, together with motor unrest, appeared, especially at night, alternating by day with periods of drowsiness during which he muttered incoherently. At night it was impossible to keep him in bed; he wandered about the ward, got in bed with other patients, crept about the floor engaged apparently in finding some lost object, entered the lavatory and laid out towels and cloths as though in the act of folding or pressing clothes. When asked what he was doing he retorted that he was a tailor and had to do his work. The blood showed a leukocyte count of 12,000 . The urine showed a trace of albumin and a few finely and coarsely granular casts.

Examination.-At the neurologic examination, April 1, 1920, the patient was found lying relatively quiet in bed with no indication of the nocturnal tendency to irrepressible agitation. He did present, however, frequent facial twitchings and contortions and almost constant and definitely choreiform movements in the hands and fingers as well as in the lower extremities. There were no gross displacements of the trunk nor coarse and ample movements at the larger joints of the extremities, but one finger after another was flexed, extended or abducted, the hand flexed, extended or supinated, the knee adducted or abducted, the foot flexed and adducted or extended and the toes similarly displaced. Otherwise he looked drowsy and apathetic and his facial expression was distinctly masklike. The eyes follawed the finger in all directions and no ophthalmoplegia could be detected, although at rest a slight divergent strabismus was evident, perhaps more marked on the right side. The pupils were small and reacted sluggishly to light and convergence efforts. The imperfect elevation of the upper lip suggested a faint paresis of the facial nerves. The tongue protruded well and without initial deviation, although it was constantly moved about, presumably participating in the general choreiform instability. There was no motor paralysis in the trunk and extremities, but the grasp was decidedly weakened, more so on the right side. The tendon reflexes were all present but hypo-active. Neither the Babinski nor Oppenheim phenomenon was present. The cremasteric and abdominal reflexes were not obtained. The sphincters were perfectly controlled. When the patient assumed the sitting or erect posture he presented a distinct intention tremor of the head. The gait was not characteristic although the patient occasionally took uneven steps and on two or three occasions showed a slight titubation. His speech was dysarthric, but by reason of his foreign birth and limited education it was difficult to interpret correctly this particular detail. At times rapid utterances were followed by slow, hesitating, almost stuttering enunciation. He exhibited amnesia for both remote and recent events, but especially for the latter, was not clearly oriented but never appeared antagonistic in the day time and obeyed all requests during the course of examination. The blood Wassermann reaction was negative. The spinal fluid was clear, gave a cell count of 25 per cubic millimeter and a strongly positive globulin reaction by Pandy's method; the Wassermann reaction was negative with 0.5 c.c. The colloidal gold reaction gave the interesting curve 5555421000 . 
Course--April 3, 1920, the patient was transferred to the psychopathic pavilion as he no longer could be managed in the general ward. From this time on he exhibited progressively more striking changes in the mental sphere; he continued to wander about at night and to get in bed with other patients. One dominant idea seemed persistently to absorb his mind and command his activities and this was the necessity of pursuing his usual work as a tailor. He repeatedly stated that he was working at his trade and this imperative concept probably explains the fact that he constantly was found busily engaged in definite tasks with his bed clothes which he either spread out carefully on the floor, wound about his person or tied up in bundles. At other times, however, his aberrations migrated into other channels and he ran about the rooms talking incoherently or laughing and singing as though in jovial mood. Night after night he crept out of bed and could not be induced to return to it, although only once did he develop any tendency to wild delirium and opposed strong resistance to physical restraint. On one occasion he was found sitting beside his bed muttering to himself in a low tone and when asked why he did not go to bed he stated that there was no place for him, the bed was filled with babies, he could see, feel and hear them, he knew they were not his own children, but of course he would not disturb nor harm them. This state of affairs continued without much change during two weeks, but the patient was nevertheless losing ground physically as well as mentally despite the fact that he ate his meals without urging and even seemed to relish his food. During this period his temperature oscillated between 99 and $101 \mathrm{~F}$., he slept less and less even in the day time, showed increasing motor weakness, looked haggard and utterly exhausted and betrayed evidence of mental depression. His muscular twitchings and choreiform movements rather decreased in severity and his speech became almost unintelligible. Otherwise the neurologic findings were not materially altered. Lumbar puncture done April 6 gave again a perfectly clear spinal fluid which showed a cell count of 23 per cubic millimeter, a well marked globulin reaction by the Pandy and Noguchi methods and a negative Wassermann reaction with 1 c.c. The colloidal gold curve this time was faintly marked and not characteristic. Another examination of the spinal fluid, April 12, showed the cell count to be 150 (?) per cubic millimeter and the globulin reaction still strongly positive.

During the week of April 18 an erythema appeared on the lateral aspect of the right arm and rapidly became infected owing to persistent scratching by the patient so that evidence of a complicating lymphangitis soon made it imperative to keep hot packs over the arm. As the patient constantly pulled off the dressings it became necessarry to keep him in bed and restrain the arms. He rebelled against this measure, but his increasing motor weakness did not enable him to oppose much resistance. The jerky restless movements which had previously diminished in severity again appeared in much accentuated form and could not be interpreted as efforts to get out of bed as there were no coarse truncal displacements nor any attempts to place the unbound lower extremities on the floor. The twitchings consisted of constantly varying movements of the hands and fingers as well as of the feet and toes, together with adduction and abduction of the knees; they were quick, jerky and irregular movements quite similar to those of chorea. The patient was sleepless, muttered a great deal, but his speech had become absolutely unintelligible. April 24, his physical condition remained practically unchanged although he exhibited a new mental attitude, seemed morbidly jovial, amused by our questions and tests and repeatedly laughed aloud for several seconds as though in derision of the 
situation. He obeyed simple orders such as showing the tongue or squeezing the hand, but could not be induced to follow the finger with the eyes nor to relax the limbs for estimation of the reflexes. The whole body was rigid and cold, the temperature subnormal, the. pulse rapid, ranging between 110 and 130, the tongue heavily coated and the sphincters apparently incontinent. The pupils were moderately wide, but responded actively to light, the tendon reflexes were not elicited, owing presumably to the rigidity. Despite this alarming state of affairs the patient continued to take nourishment satisfactorily and held his ground without much further change until May 5, when he began to show distinct evidence of substantial amelioration. He became more quiet, exhibited less confusion and disorientation, articulated more distinctly and the jerky twitchings of the extremities greatly subsided. A complete neurologic examination practiced May 8 showed a surprisingly excellent condition. The patient answered questions readily, executed commands promptly, appeared perfectly oriented as regards time, place and person, correctly estimated the length of his stay in the hospital, conversed intelligently about his family, his occupation and the onset of his illness and asked how long it would be before he would be allowed to go home. He still exhibited slight jerky twitchings in the hands, knees and feet, but was no longer rigid and could execute all the ordinary movements with the arms and legs. The whole right side seemed slightly weaker than the left and the tendon reflexes on that side were appreciably more active. There was neither Babinski nor Oppenheim phenomenon. The facial musculature on both sides was paretic, but ocular excursion was excellent in all directions, there was no ptosis and the pupils reacted actively to light and accommodation. Speech was still dysarthric but quite intelligible. Sphincteric control was perfect. His temperature had remained normal for ten days and he could now sleep relatively well.

\section{SUMMARY OF CHOREOPSYCHOTIC SYNDROME}

'This group of cases, like the preceding series, was characterized by a minimum display of the ordinary and more familiar manifestations of epidemic encephalitis. Decided somnolence or relative lethargy was present only in Case 5 and in no case was well marked ophthalmoplegia observed although partial ptosis was noted in two (Cases 4 and 6). Some degree of facial weakness, however, existed in all. The most striking features in this series were the choreiform jerkings and the persistent psychic disturbances. While the twitchings cannot be said to have been absolutely identical with those seen in classic instances of Sydenham's and Huntington's chorea, they were more closely analogous to them than to any other form of hyperkinetic disorder. These spasmodic movements, for the most part at least, were neither coarse tremors, rhythmic myoclonia nor jactitations, but consisted of rapid, jerky, irregular and incoordinate displacements almost never occurring in more than one extremity at a time and for which the term "choreiform twitchings" appears to be the only appropriate designation. The psychotic manifestations included marked and persistent mental confusion, disorientation for time and place, hallucinatory delirium, more particularly nocturnal and delusional states apparently originating in 
part from the hallucinations. It is a notable fact that in every case the hallucinations and delusions were intimately related to the usual work or occupation of the patient, who seemed totally enslaved by the fixed idea of the necessity of pursuing his habitual activities. Case 6 . is particularly interesting by reason of the remarkably accurate mimicry of the classic features of general paresis. Two of the four cases in this series terminated fatally, a point emphasized by others who have reported more or less analogous types of epidemic encephalitis.

\section{CONSIDERATIONS ON PATHOGENESIS}

The choreo-athetoid and choreopsychotic syndromes illustrated by the foregoing series of cases serve to substantiate the claim that in epidemic encephalitis the distribution of the lesions does not always limit itself to the mesencephalon and adjoining areas of the brain, and they likewise furnish an opportunity for tentative localizations in more minute or circumscribed fields within the massive area embraced by the striothalamotegmental region. While no positive conclusions can be formulated regarding the exact distribution of the lesions in the absence of satisfactory necropsies, an attempt at theoretic localization may not be unjustified on the ground that some of the symptoms observed have a well recognized anatomic basis and that much light has been thrown in recent years on the regional substratum of both choreo-athetoid syndromes and certain well defined mental disorders. In a valuable and instructive series of monographs, J. Ramsay Hunt ${ }^{13}$ has recently contributed some very interesting personal observations on the pathology of the corpus striatum, particularly with reference to the pathogenesis of paralysis agitans, and at the same time he has collected and carefully analyzed most of the previously accumulated data bearing on such affections as athetosis, Huntington's chorea, progressive lenticular degeneration and allied syndromes. He concluded that there were two syndromes resulting from elective cellular degenerative changes in the corups striatum, namely chorea and paralysis agitans. While there is great fear that the lenticular nucleus, like the endocrine glands, is being unduly called on to assume the pathogenesis of all that remains obscure in the domain of neurologic interpretation, there can be no doubt that chorei-

13. Hunt, J. Ramsay: Progressive Atrophy of the Globus Pallidus (Primary Atrophy of the Pallidal System): A Contribution to the Functions of the Corpus Striatum, Brain 40:58 (May) 1917; Tr. Am. Neurol. Assn., 1917; The Efferent Pallidal System of the Corpus Striatum: A Consideration of Its Functions and Symptomatology, Tr. Am. Neurol. Assn., 10, 1917; Primary Atrophy of the Pallidal System of the Corpus Striatum, Presented at a meeting of the Assn. Am. Phys., 1917; Clinical Types of Paralysis Referable to the Pallidal System of the Corpus Striatum, Tr. Assn. Am. Phys., 1918. 
form and athetoid disturbances are intimately related to minute lesions either within or bordering on the caudate and lenticular nuclei. Several years ago Marie and Lhermitte ${ }^{14}$ reported several cases of Huntington's chorea in which they found chronic leptomeningitis, atrophy of the frontal lobes, dilatation of the lateral ventricles and massive atrophy of the corpora striata, of the putamen more particularly. These ganglions together with the frontal cortex presented well marked degenerative changes in the nerve cells, hyperplasia of the glia tissue and definite alterations of the vascular system. Marie and Lhermitte attributed the mental disorder to the meningocortical lesions, and the choreiform manifestations to the changes found in the corpora striata. Reasoning by analogy and having in mind what has already been established regarding the distribution of the lesions in epidemic encephalitis, it is possible to furnish a plausible explanation for the symptomatic groupings observed in the present series of cases. It is true, of course, that in epidemic encephalitis the lesions are too diffuse to warrant precise deductions, but if we assume, as seems perfectly legitimate, that the subacute or chronic course of some cases is due to slowly evolving changes, there is no reason why the meningeal congestion, adventitial lymphocytic infiltrations, interstitial edema, multiple hemorrhagic extravasations and cellular disorganizations. occurring in this disease cannot give rise, according to the focal predominance of the lesions, in one case to a combination of pronounced psychotic disturbance and choreiform jerkings, and in the other case to the progressive development of well characterized athetosis or hemiathetosis. In the first instance we have in a certain measure an approach to chronic degenerative chorea differing from it of course by reason of the more abrupt development of the symptoms, the rather dissimilar physiognomy of the psychic component and the favorable or else rapidly fatal outcome. In the second case we have a clinical picture which resembles to a remarkable degree the classic examples of athetosis. It remains to be seen, however, whether such syndromes, choreoathetoid and choreopsychotic, apparently transitory or recoverable, do not recur, and whether those which fail to clear up are not eventually transformed into perfectly typical instances of the characteristic neurologic affections which they already so closely imitate.

At any rate the association of slight hemiparesis, dysarthria and disorders of emotional control with the choreo-athetoid manifestations justifies the assumption that the lesions border on the internal capsule, the absence of objective sensory losses and hemianopsia. largely warrants the elimination of serious thalamic involvement and in this way

14. Marie and Lhermitte: Les lésions de la chorée chronique progressive, Ann. de méd., No. 1, 18, 1914. 
an intralenticular or paralenticular localization asserts itself as the probable underlying cause of the spasmodic choreiform and athetoid disturbances. Thus, the pathology of epidemic encephalitis serves to confirm in a certain measure-the actually favored hypothesis regarding the striatal origin of choreic and athetoid spasms. Similarly, a predominating implication of the meninges and cortex of the frontal regions is believed to be responsible for the psychotic forms of epidemic encephalitis as well as for the psychotic components incorporated in the other clinical types of the disease, whether of the choreopsychotic or the usual midbrain type. This supposition is in keeping with the prevailing conception of the physiology and pathology of the cerebral cortex and finds additional confirmation in the strikingly paretic type of mental disturbance observed in Case 6 . It has been known for a long time that in general paresis the maximum corticomeningeal changes are distributed over the frontal regions of the cerebral hemispheres.

The occurrence of choreopsychotic and choreo-athetoid syndromes as clinical types or sequelae of epidemic encephalitis, coupled with the positive knowledge of chronic forms of this disease, opens up a wide field for retrospective meditation on the genesis of a number of diseases concerning which we still remain quite ignorant. One would think, in the first place, of the various known diseases which some of the clinical types of epidemic encephalitis more or less completely simulate, i. e., athetosis, paralysis agitans and even Huntington's chorea and Friedreich's ataxia. Despite the fact that both of the latter conditions are regarded as hereditary or familial diseases, absolutely identical clinical complexes are not uncommonly observed in which all evidence of familial character is wanting. Likewise to be mentioned in this connection are multiple sclerosis, hypothalamic and dysglandular syndromes and perhaps even dementia praecox and other psychoses. It is puerile, of course, to suppose that epidemic encephalitis is a new disease ; only our knowledge of its epidemic occurrence is new and it is. extremely probable that, like poliomyelitis, the disease has existed in sporadic form for centuries. This consideration may be helpful in our retrospective endeavor to supply a logical basis for the development of the various diseases named in the foregoing and which neurologists have been observing for years in more or less fully constituted form but without definitely ascertainable notions regarding the far remote initial disturbances.

A point of particular interest in connection with the symptomatology of epidemic encephalitis is the genesis of the variations in the clinical picture and consequently the mechanism underlying the regional variations in the focal predominance of the lesions. In an article on polio- 
myelitis, ${ }^{15}$ I have previously expressed the opinion that the localization of organic cerebrospinal disease is not entirely accidental but on the contrary largely conditioned by predisposing functional exhaustion of certain areas or cell groups resulting either from prolonged physiologic hyperactivity or from antedating trauma or peripheral disease affecting the related innervation zones. This assumption implies that the natural defensive reactions at such levels are at low ebb and consequently that the fiber tracts or cell complexes concerned, offering inadequate resistance on the advent of a cerebrospinal infectious invasion, succumb first and most severely. The resulting clinical manifestation may be either paralytic or irritative according to the level at which the neuron is disabled and according to the character of the lesion as well as to the degree of involvement. This conception may not have the merit of originality and is doubtless shared by many others, but it is nevertheless a fact that such an interpretation has sufficient practical significance to warrant greater emphasis than it actually receives either in standard textbooks or in current literature. A case in point is supplied by the observation of a patient recently. examined who presented a poliomyelitic type of epidemic encephalitis.

The case was that of a woman employed in a department store, who was constantly called on to lay on the counters merchandise stored away in a cellar. Several times a day she was obliged to lift or pull up a trap door in order to get the merchandise. March 20,1920, she developed general pains, fever of mild degree, unusual somnolence and within a period of forty-eight hours there appeared a paralysis of the right upper extremity. When seen, April 2, it was found that the paralysis was not total, involved particularly the deltoid, spinati and pectoralis major and to a lesser extent the brachialis anticus, biceps, and supinators. The paralysis was atonic with much weakened tendon reflexes in the arm and poor responses to the faradic current. The right lower extremity was somewhat rigid and exhibited increased tendon jerks. This was clearly due to a lesion in the anterior horn of the cord at the level of the fifth and sixth cervical segments with infiltration of the outlying lateral column. When seen again, May 5, all that remained of these symptoms was a much weakened grasp and inability to abduct and elevate the arm much above the horizontal.

Cases possessing similar demonstrative value have been frequently observed. Bassoe 9 recently reported a case illustrating the "meningoradicular" type of encephalitis in which a right sided facial paralysis of the peripheral type was noted in a patient who had had a facial paralysis on the same side three years before. In the present series of cases it is not particularly difficult to establish the etiologic relationship of occupational strain to the salient manifestations of the clinical picture. The traveling salesman (Case 1) who constantly

15. Archambault: The Haematogenous Invasion of the Cerebro-Spinal Axis in Poliomyelitis, Alienist and Neurologist 39:34 (Jan.) 1918. 
hurried to make train connections, impatiently stepped back and forth watching out for late arrivals, indulged at all times in hasty conversation and strove invariably to present a cheerful and winning countenance to all, developed restless movements in the legs, dysarthria and facial contortions suggestive of efforts at distant accommodation. The cigarmaker (Case 2), who spent the major portion of each day seated with his fellow workers at a long table and who constantly turned his head to the right and to the left in conversing with his companions while busily engaged in trimming and rolling tobacco leaf, developed alternating flexor and extensor athetoid movements of the head and choreo-athetoid movements in the right forearm and hand. Regarding the patient figuring as Case 3 , relevant muscular strain was apparently not concerned in the genesis of the spasmodic disorder, but, as was previously stated, the woman had had chorea several years before. The psychotic cases with one exception were observed in highly neurotic individuals of Jewish or Latin extraction who were known to have persistently worked either under considerable mental strain or overtime with altogether insufficient sleep and little opportunity for relaxation. That all of these patients continued during their illness the psychic labor associated with their usual activities was plainly revealed by the content of their hallucinations and delusions. With these several recent observations at hand and many analogous experiences in the past, one cannot help ascribing to this same factor of functional hyperactivity and focal exhaustion the preponderant involvement of the mesencephalon in epidemic encephalitis and the resulting frequency of lethargy and oculomotor paralysis. Probably never before have people slept as little and used their eyes as much as they have within the last five years. While the soldiers in the trenches were constantly on the lookout for bombs, shells and missiles of all kinds as well as for airplanes and advancing enemy detachments, the people at home hardly made a less heavy demand on the ocular musculature in persistently working overtime at the various tasks and industries directly related to the pursuit of the war. Never before have people in all walks of life so diligently read the morning and evening dailies, so frequently riveted their upturned eyes on war bulletins, so eagerly watched the evolutions of high soaring planes or so patiently stood before public buildings straining neck and eyes to get a glimpse of distinguished visitors and returning heroes. Let us now add to all this the very significant part played by motion picture theaters which are steadily cropping up everywhere in greater numbers and drawing ever increasing throngs. It is not that any one of these factors in itself has exerted a prejudicial influence but the combination of all of them operating over a protracted period has certainly implied fairly sustained hyperfunction of the motor nuclei of the midbrain 
and it is no exaggeration to state that at no time in the history of the human race have the accommodation mechanism and the associated movements of the eyeballs been brought more actively and persistently into play. It has been definitely_shown that among the ocular symptoms of epidemic encephalitis by far the most common disturbances have been represented by ptosis, weakness of convergence with paralysis of accommodation and paresis of the associated movements of the eyeballs, more particularly of vertical excursion. Is it not infinitely more logical to interpret such distinctive ocular palsies as the outcome of the selective muscle strain emphasized in the foregoing than to attribute them to a supposed specific affinity of the encephalitis virus for the ganglionic complexes of the mesencephalon?

\section{- CONCLUSIONS}

1. Epidemic encephalitis is characterized by a remarkably protean symptomatology dependent on regional variations in the focal predominance of the underlying histologic changes.

2. Aside from the usual and familiar clinical picture of midbrain involvement, the disease may yield a number of clinical types closely resembling several well-known affections of the nervous system such as paralysis agitans, poliomyelitis, polyneuritis, cerebellar sclerosis, etc. The combination of choreiform twitchings and acute psychotic disturbance as the salient manifestations is not uncommon and choreoathetoid or frankly athetoid syndromes may be observed as sequela of the disease.

3. The occurrence of a chronic or relapsing form of epidemic encephalitis may prove to have an important bearing on the origin of some of the affections which the clinical types of the disease simulate and of other ill understood conditions such as multiple sclerosis, dysglandular syndromes and certain psychoses.

4. Variations in the symptomatology of epidemic encephalitis are possibly due to individual variations in the relative susceptibility of different levels of the cerebrospinal axis and it is believed that regional exhaustion resulting from prolonged physiologic hyperactivity or from previous disease is an important factor in the localization of the lesions produced by infectious invasions of the central nervous system. 